
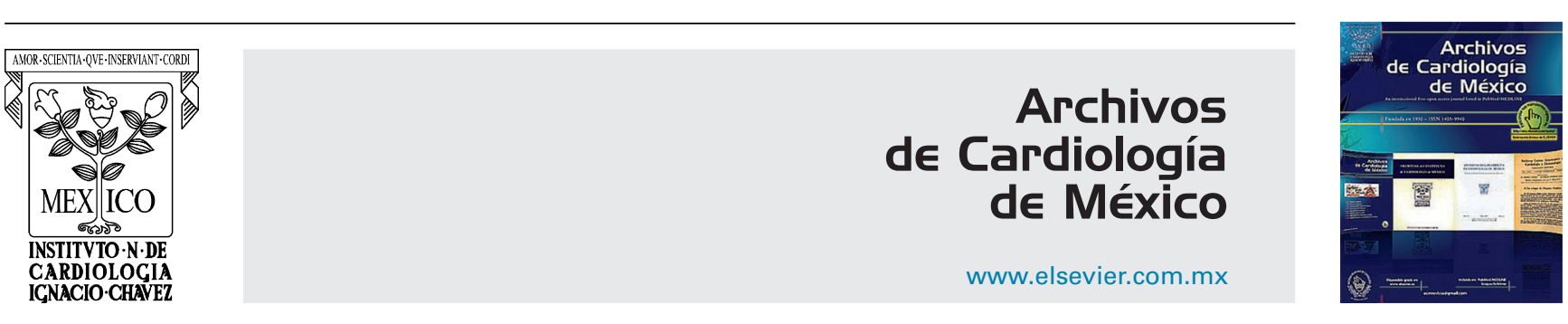

INVESTIGACIÓN CLÍNICA

\title{
Análisis demográfico de una clínica de cardiopatías congénitas del Instituto Mexicano del Seguro Social, con interés en el adulto
}

\author{
Horacio Márquez-González ${ }^{a, *}$, Lucelli Yáñez-Gutiérrez ${ }^{a}$, Jimena Lucely \\ Rivera-May ${ }^{\mathrm{b}}$, Diana López-Gallegos ${ }^{\mathrm{a}}$ y Eduardo Almeida-Gutiérrez ${ }^{\mathrm{c}}$
}

a Clínica de Cardiopatías Congénitas, Hospital de Cardiología, Centro Médico Nacional Siglo XXI, IMSS, Ciudad de México, México

${ }^{\mathrm{b}}$ Facultad de Medicina de la ANAHUAC, Ciudad de México, México

' Dirección de Investigación y Educación, Hospital de Cardiología, Centro Médico Nacional Siglo XXI, IMSS, Ciudad de México, México

Recibido el 24 de abril de 2017; aceptado el 27 de septiembre de 2017

\author{
PALABRAS CLAVE \\ Enfermedades \\ congénitas del \\ corazón; \\ Embarazo; \\ Adulto; \\ Pediátrico; \\ México
}

\begin{abstract}
Resumen
Introducción: Las cardiopatías congénitas (cc) en México se presentan con una cifra estimada entre 18,000-20,000 nuevos casos por año. La mayor parte de los centros que atienden a estos enfermos son exclusivamente pediátricos y el Instituto Mexicano del Seguro Social (IMSS) cuenta con una clínica que atiende a todas las edades.

Objetivo: Analizar los aspectos demográficos de una clínica de CC del niño y del adulto del IMSS.

Métodos: De 2011 a 2016 se realizó un estudio transversal de la clínica de CC de un hospital de tercer nivel del IMSS, incluyendo todos los pacientes con cardiopatía estructural confirmada de reciente diagnóstico. Se registró el sexo, la edad, la entidad federativa de referencia, el antecedente de embarazo y el tratamiento. Se dividió a la población en rangos de edades $(<2$ años, 2.1-6 años, 6.1-10 años, 10.1-17 años y > 17.1 años). Se empleó estadística descriptiva y prueba de $\chi^{2}$ en las variables cualitativas.

Resultados: Tres mil cuatrocientos ochenta y tres enfermos con CC (relación hombre:mujer, $0.8: 1.2)$, las cardiopatías acianógenas de flujo pulmonar aumentado son el grupo más grande (47.2\%); $25.6 \%$ fueron adultos y $35 \%$ de las mujeres con antecedente de al menos una gesta. En general la cardiopatía más frecuente fue la comunicación interauricular. La entidad federativa con mayor frecuencia de adultos de reciente diagnóstico fue Chiapas (33.82\%); el 7\% no fueron candidatos a ningún tratamiento por complicaciones inherentes a la cardiopatía.
\end{abstract}

\footnotetext{
* Autor para correspondencia. Servicio de Cardiopatías Congénitas, Hospital de Cardiología, Centro Médico Nacional Siglo XXI, Instituto Mexicano del Seguro Social, Av. Cuauhtémoc 330, C.P. 06720, Ciudad de México, México. Teléfono: (55)56276900 ext. 22203.

Correo electrónico: horacioinvestigacion@hotmail.com (H. Márquez-González).
} 


\section{KEYWORDS}

Congenital heart

diseases;

Epidemiology;

Pregnancy;

Adult;

Pediatric;

México
Conclusiones: Existe un diagnóstico tardío de la atención de las CC en la etapa adulta. Es necesario crear un registro nacional para promover nuevas políticas de salud y distribución de recursos destinados a estos pacientes.

( 2017 Instituto Nacional de Cardiología Ignacio Chávez. Publicado por Masson Doyma México S.A. Este es un artículo Open Access bajo la licencia CC BY-NC-ND (http://creativecommons. org/licenses/by-nc-nd/4.0/).

Demographic analysis of a congenital heart disease clinic of the Mexican Institute of Social Security, with special interest in the adult

\begin{abstract}
Introduction: Congenital heart disease (CHD) has an incidence of 8-10 cases per 1000 live births. In Mexico, there are 18,000-20,000 new cases per year. Most tertiary care centers for CHD attend only pediatric population; the Mexican Institute of Social Security (IMSS) has a clinic that attends pediatric and adult population.

Objective: To analyze the demographic aspects of the CHD clinic of IMSS.

Methods: From 2011 to 2016 a cross-sectional study of the CC clinic of a third level hospital of the IMSS, including all patients with confirmed structural heart disease of recent diagnosis was carried out. The sex, age, reference entity, antecedent of pregnancy and treatment were registered. The population was divided into age ranges (<2 years, 2.1-6 years, 6.1-10 years, 10.1-17 years and $>17.1$ years). Descriptive statistics and $\chi^{2}$ test were used in qualitative variables.

Results: 3,483 patients with CHD (male:female ratio, 0.8:1.2) were included. Increased pulmonary flow acyanogenic cardiopathies were the most frequent CHD group (47.2\%), with atrial septal defect being the most frequent diagnosis overall; $25.6 \%$ were adults and $35 \%$ of women with a history of pregnancy. Chiapas was Federal entity with greater proportion of patients diagnosed in the adult stage (33.82\%); $7 \%$ were not candidates for any treatment for complications of the disease.

Conclusions: CHD is a late diagnosis; it is necessary to create a national register to promote new health policies and a rational distribution of resources for these patients.

(c) 2017 Instituto Nacional de Cardiología Ignacio Chávez. Published by Masson Doyma México S.A. This is an open access article under the CC BY-NC-ND license (http://creativecommons. org/licenses/by-nc-nd/4.0/).
\end{abstract}

\section{Introducción}

En el mundo la incidencia estimada de las cardiopatías congénitas (CC) es de 4-5 por cada 1000 nacimientos $^{1}$. En México, no existen bases de datos que registren de forma sistematizada su frecuencia, por lo que el cálculo se basa en una prevalencia estimada de 8-10 por cada 1000 recién nacidos (RN), y extrapolado a la natalidad, se esperan de 18,000 a 20,000 casos nuevos por año² .

El avance en el conocimiento de las técnicas quirúrgicas, los métodos de perfusión y la atención perioperatoria ha permitido que la sobrevida actual a 45 años sea del $85 \%$, sin embargo, se precisa de un sistema de atención temprano que permita que las cirugías sean realizadas en las primeras etapas de la vida ${ }^{3}$.

En México, la realidad es que existe una centralización de los hospitales con el recurso humano y tecnológico para el tratamiento de estos enfermos, en 2006, había 9 centros hospitalarios ( 7 de ellos en la Ciudad de México) con las características para realizar cirugías en pacientes pediátricos. En la actualidad se espera que existan 21 hospitales distribuidos de acuerdo a la natalidad inferida o pronosticada en el territorio nacional ${ }^{4}$.
El incremento en la sobrevida y la modificación de la historia natural ha permitido que el enfermo con CC alcance la edad adulta y no sea exclusivo de la atención pediátrica. Esta transición demográfica demanda nuevos retos para diagnosticar y tratar las enfermedades adquiridas como cardiopatía isquémica, lesiones valvulares, arritmias cardiacas, insuficiencia cardiaca o enfermedad vascular pulmonar ${ }^{3}$. Por ello en países del norte de Europa y EE.UU. se establece la necesidad de crear las clínicas de transición que implican una inclusión multidisciplinaria de especialistas que permiten el seguimiento integral de estos pacientes ${ }^{5}$. Esta realidad no se ha visto reflejada en los programas de atención de los centros de tercer nivel de nuestro país, ya que existe una pérdida del seguimiento del paciente reparado de CC en la etapa pediátrica al momento de que es egresado de estos hospitales. En el Instituto Mexicano del Seguro Social (IMSS), existe en la Ciudad de México un hospital de tercer nivel con una clínica de CC que atiende a este tipo de enfermos desde la infancia hasta la edad adulta. La experiencia de este tipo de centros, aplicado a la realidad actual del país permite ofrecer un panorama de la situación actual.

El objetivo primario de este trabajo fue analizar la frecuencia de las CC atendidas en la «Clínica de Cardiopatías 
Congénitas del niño y del adulto» de un hospital de tercer nivel del IMSS.

\section{Métodos}

Se realizó un estudio transversal descriptivo y analítico, que fue presentado y aprobado por el comité local de ética en investigación. En el periodo comprendido entre 2011 a 2016 se incluyeron todos los enfermos con diagnóstico de certeza de CC atendidos en la clínica en cualquiera de las siguientes áreas: hospitalización, consulta externa y área de ecocardiografía pediátrica. Fueron excluidos del análisis los enfermos con cardiopatía adquirida (endocarditis, miocarditis, fiebre reumática, enfermedad de Kawasaki, enfermedad de Takayasu, hipertensión pulmonar primaria y secundarias a isquemia coronaria), arritmias no asociadas a CC y con tratamiento quirúrgico cardiológico de cualquier tipo.

La información sobre la población de estudio se recabó de la siguiente manera:

- Numeradores: todos los enfermos con CC confirmada atendidos en el hospital sede del estudio.

- Denominadores: número total de pacientes que fueron confirmados de CC en cualquiera de las áreas mencionadas.

- Variables de persona: sexo, edad, entidad federativa de referencia de los pacientes. Sobre la información de los procedimientos a los cuales se consideraron candidatos se obtuvo del documento de sesión médico-quirúrgica. Se agregó un rubro sobre el antecedente de embarazo en las mujeres mayores de 17 años, que fue obtenido a partir de la hoja de trabajo social y de la historia clínica.

Para la codificación de la información se capacitaron 2 cardiólogos pediatras y un médico pasante de servicio social, la concordancia intra e interobservador para llenar la hoja de datos el valor kappa $>0.80$.

Las fuentes primarias de información que conformaron la base de datos clínica fueron: censos diarios, expediente médico (físico y/o electrónico), reportes de estudios de gabinete (ecocardiograma, tomografía cardiaca y cateterismo cardiaco).

Cuando la información fue totalmente recabada, con el apoyo del departamento de trabajo social y de asistentes médicas se codificaron los lugares de origen a partir del apartado correspondiente del número de seguridad social.

Las CC fueron clasificadas de acuerdo a su presentación clínica en:

1. Acianógenas de flujo pulmonar normal: que incluye coartación aórtica (CoA), estenosis subvalvular, valvular o supravalvular aórtica congénita, interrupción del arco aórtico, estenosis subvalvular, valvular o supravalvular pulmonar.

2. Acianógenas de flujo pulmonar incrementado: comunicación interauricular $(\mathrm{CIA})$, comunicación interventricular (CIV), persistencia del conducto arterioso (PCA), conexión anómala total o parcial de venas pulmonares, ventana aortopulmonar

3. Cianógenas de flujo pulmonar disminuido: tetralogía de Fallot (TF), atresia pulmonar con CIV $(\mathrm{AP}+\mathrm{CIV})$, atresia pulmonar sin CIV (AP sin CIV), anomalía de Ebstein (AE), atresia tricuspídea (AT)

4. Cianógenas de flujo pulmonar incrementado: transposición de grandes arterias (TGA), canal auriculoventricular, tronco común (TC), doble vía de salida del ventrículo derecho (DVS-VD)

Con la edad al momento del diagnóstico se dividió al total de la población en 5 categorías de acuerdo a la clasificación de las edades pediátricas:

1. Menores de 2 años (lactantes)

2. 2.1-6 años (preescolares)

3. 6.1-10 años (escolares)

4. 10.1-17 años (púberes y adolescentes)

5. Mayores de 17.1 años (por situaciones administrativas, en este centro médico correspondiente al IMSS se consideran adultos a partir de esta edad)

Análisis estadístico: Para el análisis descriptivo se emplearon en variables cualitativas frecuencias absolutas y porcentajes. Para determinar las diferencias entre los grupos de edad se empleó la prueba de $\chi^{2}$. El programa estadístico empleado fue SPSS ${ }^{\circledR}$, versión 20 para MAC.

\section{Resultados}

Fueron enviados a evaluación en el periodo del estudio 5,780 pacientes con sospecha de CC, de los cuales 3,483 se confirmaron $(1,520$ sin cardiopatía estructural y 777 con cardiopatía adquirida); 1,926 (55\%) fueron mujeres y la relación hombre:mujer fue de $0.8: 1.2$, la distribución entre grupo de edad y el sexo (tabla 1).

La distribución por grupo de edad fue la siguiente: menores de 2 años en 447 (12.83\%), 2.1-6 años en 768 (22.05\%), de 6.1-10 años en 741 (21.27\%), 10.1-17 años en 634 (18.20\%) y mayores de 17.1 años en 893 (25.64\%).

La distribución según la clasificación clínica de las CC fue: cardiopatías acianógenas de flujo pulmonar incrementado en 1,645 (47.23\%) pacientes; cianógenas de flujo pulmonar normal en 767 (22.02\%), acianógenas de flujo pulmonar normal en $173(4.97 \%)$ y $173(4.97 \%)$ pacientes con cardiopatías cianógenas de flujo pulmonar normal.

En el caso de las 558 (28.64\%) mujeres adultas, 100 (18\%) fueron diagnosticadas de CC durante el embarazo y 95 (17\%) ya se conocían portadoras, sin tratamiento, sin método de planificación familiar y se embarazaron.

En general, la CC más frecuente fue la CIA en 853 (49\%) sujetos y también en la mayoría de los grupos de edad,

Tabla 1 Distribución de las cardiopatías congénitas por grupo de edad y sexo

\begin{tabular}{|c|c|c|c|c|c|}
\hline \multirow[b]{2}{*}{$<2$ años } & \multicolumn{2}{|c|}{ Hombre (\%) } & \multicolumn{2}{|c|}{ Mujer (\%) } & \multirow{2}{*}{$\frac{\text { Total }}{447}$} \\
\hline & 188 & 5.40 & 259 & 7.43 & \\
\hline 2.1-6 años & 395 & 11.34 & 373 & 10.71 & 768 \\
\hline 6.1-10 años & 341 & 9.79 & 400 & 11.48 & 741 \\
\hline 10-17 años & 298 & 8.56 & 336 & 9.64 & 634 \\
\hline \multirow[t]{2}{*}{ > 17.1 años } & 335 & 9.62 & 558 & 16.02 & 893 \\
\hline & 1557 & 44.70 & 1926 & 55.28 & 3483 \\
\hline
\end{tabular}


Tabla 2 Frecuencia y porcentaje de las cardiopatías congénitas agrupados por edad

\begin{tabular}{|c|c|c|c|c|c|c|c|c|c|c|c|c|}
\hline \multirow[t]{2}{*}{ Cardiopatía } & \multicolumn{2}{|c|}{$<2$ años } & \multicolumn{2}{|c|}{ 2.1-6 años } & \multicolumn{2}{|c|}{ 6.1-10 años } & \multicolumn{2}{|c|}{ 10-17 años } & \multicolumn{2}{|c|}{$>17.1$ años } & \multicolumn{2}{|c|}{ Total } \\
\hline & Número & Porcentaje & Número & Porcentaje & Número & Porcentaje & Número & Porcentaje & Número & Porcentaje & Número & Porcentaje \\
\hline Anomalía de Ebstein & 20 & 4.47 & 7 & 0.91 & 25 & 3.37 & 30 & 4.73 & 77 & 8.62 & 159 & 4.57 \\
\hline Atresia pulmonar con CIV & 71 & 15.88 & 99 & 12.89 & 20 & 2.70 & 17 & 2.68 & 0 & 0.00 & 207 & 5.94 \\
\hline Atresia pulmonar sin CIV & 70 & 15.66 & 80 & 10.42 & 10 & 1.35 & 0 & 0.00 & 0 & 0.00 & 160 & 4.59 \\
\hline Atresia tricuspídea & 42 & 9.40 & 12 & 1.56 & 0 & 0.00 & 0 & 0.00 & 0 & 0.00 & 54 & 1.55 \\
\hline Canal auriculoventricular & 20 & 4.47 & 15 & 1.95 & 11 & 1.48 & 8 & 1.26 & 0 & 0.00 & 54 & 1.55 \\
\hline Coartación aórtica & 23 & 5.15 & 49 & 6.38 & 85 & 11.47 & 101 & 15.93 & 99 & 11.09 & 357 & 10.25 \\
\hline Comunicación interauricular & 11 & 2.46 & 115 & 14.97 & 157 & 21.19 & 143 & 22.56 & 427 & 47.82 & 853 & 24.49 \\
\hline Comunicación interventricular & 17 & 3.80 & 108 & 14.06 & 141 & 19.03 & 89 & 14.04 & 77 & 8.62 & 432 & 12.40 \\
\hline Conexión anómala de venas pulmonares & 15 & 3.36 & 4 & 0.52 & 3 & 0.40 & 3 & 0.47 & 8 & 0.90 & 33 & 0.95 \\
\hline Doble discordancia & 1 & 0.22 & 1 & 0.13 & 3 & 0.40 & 15 & 2.37 & 19 & 2.13 & 39 & 1.12 \\
\hline Doble vía de salida del VD & 17 & 3.80 & 18 & 2.34 & 18 & 2.43 & 11 & 1.74 & 8 & 0.90 & 72 & 2.07 \\
\hline Estenosis aórtica con aorta bivalva & 5 & 1.12 & 19 & 2.47 & 32 & 4.32 & 36 & 5.68 & 29 & 3.25 & 121 & 3.47 \\
\hline Estenosis mitral & 1 & 0.22 & 6 & 0.78 & 2 & 0.27 & 1 & 0.16 & 3 & 0.34 & 13 & 0.37 \\
\hline Estenosis pulmonar & 2 & 0.45 & 36 & 4.69 & 28 & 3.78 & 32 & 5.05 & 22 & 2.46 & 120 & 3.45 \\
\hline Estenosis ramas pulmonares & 0 & 0.00 & 2 & 0.26 & 1 & 0.13 & 0 & 0.00 & 0 & 0.00 & 3 & 0.09 \\
\hline Estenosis supravalvular aórtica & 0 & 0.00 & 1 & 0.13 & 3 & 0.40 & 6 & 0.95 & 2 & 0.22 & 12 & 0.34 \\
\hline Estensosis subvalvular aórtica & 2 & 0.45 & 5 & 0.65 & 7 & 0.94 & 12 & 1.89 & 12 & 1.34 & 38 & 1.09 \\
\hline Miocardiopatía hipertrófica & 2 & 0.45 & 3 & 0.39 & 10 & 1.35 & 9 & 1.42 & 12 & 1.34 & 36 & 1.03 \\
\hline Persistencia del conducto arterioso & 20 & 4.47 & 96 & 12.50 & 97 & 13.09 & 56 & 8.83 & 58 & 6.49 & 327 & 9.39 \\
\hline Tetralogía de Fallot & 68 & 15.21 & 81 & 10.55 & 79 & 10.66 & 50 & 7.89 & 32 & 3.58 & 310 & 8.90 \\
\hline Transposición de grandes arterias & 35 & 7.83 & 0 & 0.00 & 0 & 0.00 & 0 & 0.00 & 0 & 0.00 & 35 & 1.00 \\
\hline Tronco común & 2 & 0.45 & 5 & 0.65 & 1 & 0.13 & 2 & 0.32 & 0 & 0.00 & 10 & 0.29 \\
\hline Ventana aortopulmonar & 2 & 0.45 & 0 & 0.00 & 0 & 0.00 & 0 & 0.00 & 0 & 0.00 & 2 & 0.06 \\
\hline Ventrículo derecho hipoplásico & 1 & 0.22 & 6 & 0.78 & 8 & 1.08 & 13 & 2.05 & 8 & 0.90 & 36 & 1.03 \\
\hline Total & 447 & 100.00 & 768 & 100.00 & 741 & 100.00 & 634 & 100.00 & 893 & 100.00 & 3483 & 100.00 \\
\hline
\end{tabular}

CIV: comunicación interventricular; VD: ventrículo derecho. 
con excepción de los menores de 2 años que fue la AP + CIV (15.88\%) (tabla 2).

El análisis entre el grupo de edad al diagnóstico de la CC y la entidad federativa que lo refirió demostró que Chiapas y Guerrero enviaron $52.97 \%$ de los enfermos detectados en mayores de 17.1 años y discrepa con los referidos durante la etapa de lactantes de la zona metropolitana en el $73.26 \%$ (tabla 3).

En relación a los procedimientos realizados (tabla 4), en $1,493(42.87 \%)$ pacientes el defecto cardiaco se reparó mediante cateterismo intervencionista y en 1,409 (40.46\%) enfermos se realizó mediante procedimiento quirúrgico. Fueron $225(6.46 \%)$ casos que no fueron tributarios de algún procedimiento, en $176(5 \%)$ de ellos porque las cifras de presión arterial pulmonar tuvieron un resultado mayor a la tensión arterial sistémica en el cateterismo con enfermedad vascular pulmonar (resistencias pulmonares fijas al reto de óxido nítrico).

\section{Discusión}

Según el Instituto Nacional de Estadística y Geografía (INEGI), las malformaciones del sistema circulatorio representaron en 2015 la segunda causa de muerte en menores de un año y dentro de las primeras 3 en los siguientes 15 años de vida ${ }^{6}$. Torres-Cosme et al., analizaron la mortalidad neonatal con los datos del INEGI entre 1998-2013, reportando $41,717,421$ nacimientos, de los cuales se atribuyeron $50,759(24.8 \%)$ muertes a CC, con una tasa de mortalidad de 121.7 por 100,000 RN. En dicho estudio, las CC acianógenas de flujo pulmonar incrementado fueron las más frecuentes (PCA, CIV y CIA) y en segundo plano las CC cianógenas (TGA y TF). La cardiopatía más asociada a mortalidad fue la PCA y la zona centro del país (metropolitana) la que presentó mayor número de muertes ${ }^{7}$. Estos datos representan un sesgo, tomando en cuenta la distribución de los centros hospitalarios donde se atienden a estos pacientes, y además que los diagnósticos no fueron corroborados debido a que la información se obtuvo de los certificados de defunción.

La realidad en nuestro país es que el cálculo de la incidencia y prevalencia de las CC es empírico. La revisión de la literatura científica nacional de los últimos 10 años, demuestra que la distribución es heterogénea según el centro que lo reporte (tabla 5). Los artículos que analizaron la estadística de la frecuencia de CC en RN concuerdan que el grupo de cardiopatías acianógenas de flujo pulmonar incrementado son las más frecuentes, con orden variado entre la CIA, PCA y CIV, según el centro hospitalario ${ }^{8-11}$. En este trabajo dicho grupo de CC presentó una frecuencia general del 47.23\%.

Cuando se analizan los trabajos publicados sobre la experiencia en los pacientes reparados quirúrgicamente, las frecuencias se dispersan según el centro hospitalario, por ejemplo, la casuística de cirugías en cardiopatías complejas es mayor en los hospitales públicos de la Ciudad de México, comparada con otros hospitales públicos (de otras entidades federativas fuera de la zona centro) y privados ${ }^{12-14}$. Debe hacerse una referencia especial al esfuerzo de la Asociación Mexicana de Especialistas en Cardiopatías Congénitas (AMECC) que publicó la primer fase de una base de datos que concentró la experiencia de centros quirúrgicos de todos los estados del país, en un periodo de 3 años, y sumó un total

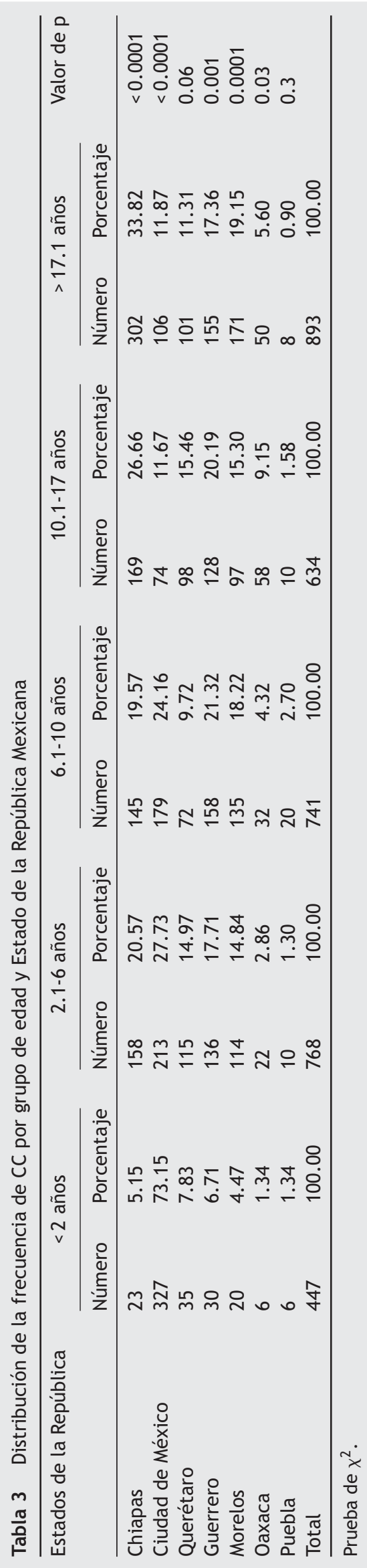


Tabla 4 Procedimientos generales realizados en la serie de cardiopatías congénitas

\begin{tabular}{|c|c|c|c|}
\hline \multirow[t]{2}{*}{ Procedimientos } & \multirow[t]{2}{*}{ Número } & \multicolumn{2}{|c|}{ Porcentaje } \\
\hline & & Dentro del grupo & Total \\
\hline Cierre por cateterismo intervencionista & 1493 & 100.0 & 42.87 \\
\hline Cierre de cortocircuitos & 870 & 58.27 & 24.97 \\
\hline Angioplastías/valvuloplastias & 483 & 32.35 & 13.86 \\
\hline Colocación de stent en $\mathrm{CoA}$ & 100 & 6.70 & 2.89 \\
\hline Otras $^{\mathrm{a}}$ & 40 & 2.68 & 1.15 \\
\hline Cierre quirúrgico del defecto septal & 570 & 100.0 & 16.37 \\
\hline Reparación total de cirugía compleja & 839 & 100.0 & 24.09 \\
\hline Restablecer continuidad VD/TAP & 502 & 59.83 & 14.41 \\
\hline Plastia del arco aórtico & 202 & 24.09 & 5.80 \\
\hline Ross-Konno & 38 & 4.52 & 1.09 \\
\hline Jatene & 11 & 1.31 & 0.32 \\
\hline Otros $^{b}$ & 86 & 10.25 & 2.47 \\
\hline Implante valvular & 245 & 100.0 & 7.03 \\
\hline Aorta & 112 & 45.8 & 3.22 \\
\hline Pulmonar & 79 & 32.2 & 2.26 \\
\hline Tricúspide & 29 & 11.8 & 0.83 \\
\hline Mitral & 25 & 10.2 & 0.72 \\
\hline Cirugías paliativas & 65 & 100.0 & 1.87 \\
\hline Fístula tipo Blalock-Taussig & 48 & 73.8 & 1.3 \\
\hline Fistulas centrales & 10 & 15.4 & 0.28 \\
\hline Fístulas veno/venosas & 7 & 10.8 & 0.2 \\
\hline Cirugía de Fontan & 46 & 100.0 & 1.32 \\
\hline Ningún procedimiento & 225 & 100.0 & 6.46 \\
\hline HAP suprasistémica & 176 & 78.3 & 5.0 \\
\hline Comorbilidades con riesgo $>$ beneficio & 22 & 9.7 & 0.63 \\
\hline Negativa de padres/tutores & 18 & 8.0 & 0.51 \\
\hline Insuficiencia cardiaca & 9 & 4.0 & 0.25 \\
\hline Total & 3483 & 100.00 & \\
\hline
\end{tabular}

CoA: coartación aórtica; HAP: hipertensión arterial pulmonar; VD/TAP: ventrículo derecho/tronco de la arteria pulmonar.

*: colocación de dispositivos para cerrar continuidad VD/TAP, cierre de colaterales aortopulmonares.

**: focalización de colaterales aortopulmonares, reconexión de colector de venas pulmonares, interposición de tubo aórtico y ampliación de ramas.

de 930 procedimientos quirúrgicos, de los cuales el cierre de CIV fue la cirugía mayor mayormente realizada en el caso del grupo de cardiopatías de flujo pulmonar incrementado, seguida de la CIA; y la reparación de TF en el caso de las cianógenas ${ }^{15}$.

En este trabajo reportamos una frecuencia de CC diagnosticadas en la edad adulta del $25.64 \%$, siendo la CIA (47\%) la más frecuente, lo que concuerda con la información publicada con el Centro Médico Nacional «La Raza» (IMSS) ${ }^{16}$, quienes también la ubican en primer puesto y refuerza con lo publicado previamente en nuestro centro ${ }^{17}$; sin embargo discrepa en la casuística de la clínica de CC del Instituto Nacional de Cardiología, que reportaron a la CIV en primer sitio ${ }^{18}$. La siguiente cardiopatía en frecuencia en nuestra serie, fue la CoA, misma que no tiene similitud con los 2 trabajos previamente referidos.

Particularmente resulta de interés que en nuestro hospital, el 35\% de las mujeres adultas presentaron antecedente de embarazo (con y sin conocimiento de ser portadoras de CC) con cardiopatía no reparada; no hay manera de equiparar estos datos con algún estudio mexicano de la misma naturaleza, sin embargo en el Instituto Nacional de Perinatología, publicó un estudio de pronóstico que evaluó la sobrevida de mujeres embarazadas con CC, que los defectos más frecuentes fueron la CIV y la $\mathrm{CIA}^{19}$. Sobre el diagnóstico fetal, el único estudio publicado corresponde al centro previamente citado, donde reportan una frecuencia de 73 fetos con CC en 10 años, en la clínica de medicina materno-fetal, reflejando que el diagnóstico in-utero, es aún en México un recurso limitado ${ }^{20}$.

Tomando en cuenta la dificultad que implica el acceso del paciente con CC a un centro especializado, el escenario de la mujer embarazada con CC en México es aún más restringido, debido a que en su mayoría estos centros son pediátricos y no cuentan con acceso directo a los servicios de gineco-obstetricia, lo que incrementa el riesgo de morbimortalidad. Es esperado que la mujer cardiópata presente complicaciones en el periparto y puerperio inmediato en el 25-30\%, y que los RN desarrollen complicaciones (prematurez, asfixia, restricción en el crecimiento, muerte) hasta en el $54 \%{ }^{21}$.

La zona de influencia del hospital de tercer nivel donde se realizó este estudio, son estados catalogados por el INEGI con el mayor rango de pobreza ${ }^{22}$, particularmente Chiapas y Guerrero mostraron una diferencia estadística al comparar el grupo de edad al momento de la referencia, es decir 
Tabla 5 Artículos de hospitales nacionales donde se refiere la frecuencia de cardiopatías congénitas en distintos grupos de edad

\begin{tabular}{|c|c|c|}
\hline Autor & Referencia & Hospital \\
\hline $\begin{array}{l}\text { Alva- } \\
\text { Espinosa }\end{array}$ & 17 & $\begin{array}{l}\text { Hospital de } \\
\text { Cardiología, } \\
\text { Centro Médico } \\
\text { Nacional Siglo xxI }\end{array}$ \\
\hline $\begin{array}{l}\text { Bermudez- } \\
\text { Alarcón }\end{array}$ & 8 & $\begin{array}{l}\text { Centro Médico } \\
\text { Nacional } 20 \text { de } \\
\text { noviembre. ISSSTE }\end{array}$ \\
\hline $\begin{array}{l}\text { Castillo- } \\
\text { Espínola }\end{array}$ & 12 & $\begin{array}{l}\text { Unidad Médica de } \\
\text { Alta Especialidad, } \\
\text { Yucatán «lgnacio } \\
\text { García Téllez» }\end{array}$ \\
\hline $\begin{array}{l}\text { Cervantes- } \\
\text { Salazar }\end{array}$ & 15 & AMECC \\
\hline $\begin{array}{l}\text { De Rubens- } \\
\text { Figueroa }\end{array}$ & 11 & $\begin{array}{l}\text { Instituto Nacional } \\
\text { de Pediatría }\end{array}$ \\
\hline Flores-Nava & 9 & $\begin{array}{l}\text { Hospital General } \\
\text { Gea González }\end{array}$ \\
\hline $\begin{array}{l}\text { Galván- } \\
\text { Román }\end{array}$ & 16 & $\begin{array}{l}\text { Hospital de } \\
\text { Especialidades, } \\
\text { Centro Médico } \\
\text { Nacional «La } \\
\text { Raza» }\end{array}$ \\
\hline $\begin{array}{l}\text { Hernández- } \\
\text { Pacheco }\end{array}$ & 19 & $\begin{array}{l}\text { Instituto Nacional } \\
\text { de Perinatología } \\
\text { «Isidro Espinosa } \\
\text { Reyes» }\end{array}$ \\
\hline $\begin{array}{l}\text { Mendieta- } \\
\text { Alcántara }\end{array}$ & 10 & $\begin{array}{l}\text { Hospital General } \\
\text { «Lic. Adolfo López } \\
\text { Mateos» y Hospital } \\
\text { Materno Perinatal } \\
\text { «Mónica Pretelini» }\end{array}$ \\
\hline $\begin{array}{l}\text { Solano- } \\
\text { Fiesco }\end{array}$ & 13 & $\begin{array}{l}\text { Hospital Central } \\
\text { Militar }\end{array}$ \\
\hline
\end{tabular}

$\begin{array}{lll}\text { Varela-Ortiz } & 14 \quad \begin{array}{l}\text { Hospital Médica } \\ \text { Sur }\end{array}\end{array}$

Vázquez- $\quad 18$ Antona

Velazquez- $\quad 20$ Torres

de Cardiología, «lgnacio Chávez»

Instituto Nacional de Perinatología

$\begin{array}{ll}\text { Año } & \text { Resultados } \\ \text { 2000-2005 } & \text { Adultos operados de la clínica de CC, las } 10 \text { pri } \\ & \text { causas fueron: CIA, 62\%; Ao. bivalva, 10.4\%; PCA, } \\ & \text { CIV, 5.2\%; diafagma subaórtico, } 3.5 \% \text {; CoA, } 3 \% ; \\ & \text { ectasia anulo-aórtica, } 2.2 \% \text {; TF, } 1.7 \% \text {; corazón } \\ & \text { univentricular, 0.6\% y CAVP, en 0.6\% } \\ 1998-2000 & \text { En 1,830 estudios ecocardiográficos en menores }\end{array}$

1998-2000 En 1,830 estudios ecocardiográficos en menores de 18 años se encontraron 1,161 con CC, los principales diagnósticos fueron: CIV, 38\%; CIA, 31\%; PCA, 26\%; Ao. bivalva, $8.8 \%$; TF, $6.2 \%$; CoA, $4.9 \%$ y canal AV, en $3.7 \%$

2011-2013 En 85 pacientes reparados de CC, 73\% fueron cardiopatías simples y $27 \%$ complejas, los principales diagnósticos fueron: PCA, 37.6\%; CIA, $11.8 \%$; CIV, $11.8 \%$; TF, $3.5 \%$ y atresia pulmonar, en $2.7 \%$

2011-2013 Registro de 930 cirugías en 880 pacientes, por las siguientes causas: CIV, 14.8\%; PCA, 10.9\%; TF, 8.1\%: CIA, $7.8 \%$; CAVP, $6.7 \%$ y CoA en $6.1 \%$

1983-1993 En 321 neonatos con CC la frecuencia fue: PCA, 48.9\%; CIV, 11.8\%; TGA, 5.9\%; atresia pulmonar, $5 \%$; síndrome de ventrículo único, $4 \%$; CoA, $3.4 \%$ y CAVP, en $2.2 \%$

2002-2007 De 22,327 neonatos, en 416 se presentaron Adultos Población

Adultos

malformaciones y 49 presentaron CC que fueron: CIA, $28 \%$; PCA, $28 \%$ y CIV en $24 \%$

2004-2006 De 9,833 estudios ecocardiográficos, 1,071 correspondiente a adultos, y la frecuencia de CC fue: CIA, 39\%; Ao. bivalva, 19\%; CIV, 14\%; PCA, 10\%; CoA, $7 \%$; estenosis pulmonar, $6 \%$; $\mathrm{AE}, 4 \%$ y TF en $2.3 \%$

2005-2010 En 40 mujeres embarazadas con CC con Mujeres cortocircuitos la frecuencia fue: CIV, 35\%; CIA, 33.5\%; embarazadas PCA, $18 \%$ y más de uno en $13.5 \%$

2006-2010 Entre ambos hospitales nacieron en el periodo 23,926 Neonatos RN vivos, 117 con CC $(7.4 \times 1000$ RN vivos $)$ y la frecuencia de CC fue: PCA, 23.7\%; CIA, 22.5\%; CIA + PCA, 19.2\%; CIV, 9.6\%; AP sin CIV, 1.7\%; TF, $1.7 \%$ y $\mathrm{AE}$ en $1.1 \%$ «lsidro Espinosa Reyes»
2006-2010 De 1,119 pacientes atendidos en el periodo en el servicio de cardiología pediátrica, en 628 (78.1\% acianógena, $21.9 \%$ cianógena) casos se corroboró CC que fueron: CIA, 28.6\%; PCA, 15.2\%; TF, 2.7\% y TGA en $1.1 \%$

2013-2015 En 20 pacientes con CC fueron intervenidos quirúrgicamente, 19 por cirugías correctivas y uno por paliativa las frecuencias fueron: CIV, 21\%; TF, 21\%; TGA, $10 \%$; CoA, $10 \%$; canal AV, $5 \%$; estenosis subaórtica, $5 \%$; CIA, $5 \%$ y CAVP en $5 \%$

2002 Fueron 651 adultos atendidos en la clínica de CC de este centro por: CIV, $18 \%$; CIA $14 \%$, estenosis aócnalrtica, 13\%; estenosis pulmonar, 9\%; AE, 9\%; TF, 9\%; CoA, $4 \%$; estenosis con CIV, $3 \%$ y atresia tricuspídea en $3 \%$

Pediátricos

Pediátricos

Adultos

1996-2006 Ecocardiogramas fetales del departamento de medicina materno-fetal; 73 pacientes de los cuales: defectos del septum $20.5 \%$, AE, $19 \%$, canal AV, $17.8 \%$, ventrículo izquierdo hipoplásico, $17.8 \%$

Ao: aorta; AE: anomalía de Ebstein; AMECC: Asociación Mexicana de Especialistas en Cardiopatías Congénitas; CAVP: conexión anómala de venas pulmonares; canal AV: canal auriculoventricular; CC: cardiopatía congénita; CIA: comunicación interatrial; CIV: comucación interventricular; CoA: coartación aórtica; PCA: persistencia del conducto arterioso; RN: recién nacido; TF: tetralogía de Fallot; TGA: transposición de las grandes arterias. 
fueron enviados en etapas pediátricas más avanzadas, y al $51.18 \%$ de los adultos diagnosticados por primera vez con $\mathrm{CC}$, lo que refleja un retraso en el diagnóstico y la atención; sobre lo anterior, Knowles et al., analizaron en Reino Unido, las características sociodemográficas de 5,300 niños con CC, reportando que no existe diferencia en la distribución y complejidad de las cardiopatías, pero sí en la calidad y rapidez de la distribución de los servicios médicos, al comparar entre razas, siendo las no caucásicas las menos beneficiadas ${ }^{23}$. Los autores reportamos que en la edad adulta, existió una mayor frecuencia de mujeres, probablemente porque el embarazo aceleró el proceso de diagnóstico y en relación a esta idea, Theran et al., hicieron una comparación entre sexos en 347 adultos con CC, encontrando que las mujeres representaron mayor necesidad de servicios, sin embargo los hombres tuvieron una frecuencia mayor en hábitos nocivos como el tabaquismo, con mayor prevalencia de complicaciones graves; en ambos grupos más del 50\% manifestaron desconocer el tipo de CC que eran portadores ${ }^{24}$. Sin embargo, Aubry y Demian reportaron que la mujer con CC, una vez que está embarazada tiene mayor frecuencia de comorbilidades ${ }^{25}$.

La información previamente analizada, revela la premura de realizar en México, un registro nacional que permita justificar la distribución de recursos humanos y materiales destinados a la atención del enfermo con CC. Un estudio reportado por Mackie et al., realizado en Canadá reportó en 22,096 adultos con CC una mayor frecuencia de reingresos y hospitalizaciones en salas de urgencias, que en el $87 \%$, requirieron de atención de subespecialistas ${ }^{26}$. La regionalización con base a la estadística, permite justificar la formación recursos en salud que ofrezcan la atención de acuerdo a las necesidades de los centros especializados para la atención de estos pacientes.

En el caso de nuestra clínica de CC, menos del 7\% no fueron tributarios a ningún tratamiento de reparación quirúrgica, lo que refleja que en la mayoría de los casos, pueden realizarse tratamientos definitivos o paliativos. No obstante, ya ha sido evidenciado en estudios de costo-efectividad, que a pesar de que el paciente con CC confiere mayor gasto, la detección oportuna puede hacer la diferencia entre la realización de un procedimiento paliativo o curativo, lo que se refleja en una mejor calidad de vida y un reintegro a la sociedad económicamente activa ${ }^{27-29}$.

Las ventajas de este estudio radican en la exposición y el análisis de la casuística de la única clínica de CC de población derecho-habiente a seguridad social que atienen a todas las edades del país.

Debe señalarse como debilidad de método, que la logística de atención en nuestro centro obliga que la atención en menores de 4 años sea otorgada en otra sede del IMSS, por lo que las cardiopatías graves del neonato no están consignadas en este análisis. Por las circunstancias ya mencionadas no es posible controlar el sesgo de referencia y las pérdidas por sesgos de mala clasificación.

Es manifiesto el hueco en el conocimiento sobre la frecuencia de CC en México y justifica la necesidad de realizar estudios multicéntricos nacionales que permitan contestar dichas preguntas.

En conclusión, existe un diagnóstico tardío de la atención de las CC en la etapa adulta. Es necesario crear un registro nacional para promover nuevas políticas de salud y distribución de recursos destinados a estos pacientes.

\section{Responsabilidades éticas}

Protección de personas y animales. Los autores declaran que para esta investigación no se han realizado experimentos en seres humanos ni en animales.

Confidencialidad de los datos. Los autores declaran que han seguido los protocolos de su centro de trabajo sobre la publicación de datos de pacientes.

Derecho a la privacidad y consentimiento informado. Los autores declaran que en este artículo no aparecen datos de pacientes.

\section{Financiación}

Los autores declaran que no hay financiación relacionada con el artículo.

\section{Conflicto de intereses}

Los autores declaran no presentar conflicto de intereses.

\section{Agradecimientos}

A la Dra. Yazmín Copado Mendoza, por su colaboración en la aportación de información y experiencia, para la elaboración de este tema.

\section{Bibliografía}

1. Hoffman JI, Kaplan S. The incidence of congenital heart disease. JACC. 2002;39:1890-900.

2. Samanek M. Congenital heart malformations: Prevalence, severity, survival, and quality of life. Cardiol Young. 2000;10:179-85.

3. Nieminen HP, Jokinen EV, Sairanen HI. Causes of late deaths after pediatric cardiac surgery: A population-based study. JACC. 2007;50:1263-71.

4. Calderón-Colmenero J, Curi-Curi PJ, Ramírez-Marroquín S. Congenital heart disease in Mexico Regionalization proposal. Arch Cardiol Mex. 2010;80:133-40.

5. Clarizia NA, Chahal N, Manlhiot C, et al. Transition to adult health care for adolescents and young adults with congenital heart disease: Perspectives of the patient, parent and health care provider. Can J Cardiol. 2009;25:e317-22.

6. INdEyG. Mortalidad. Estadísticas de Mortalidad. 2015. [consultado 13 Abr 2017]. Disponible en: http://www.beta.inegi.org. $\mathrm{mx} /$ proyectos/registros/vitales/mortalidad/

7. Torres-Cosme JL, Rolon-Porras C, Aguinaga-Rios M, et al. Mortality from Congenital Heart Disease in Mexico: A Problem on the Rise. Plos One. 2016;11:e0150422.

8. Bermudez-Alarcón J. Incidencia de las cardiopatías congénitas en el Centro Medico Nacional «20 de Noviembre», Revisión de reportes del servicio de ecocardiografía 1998-2000. Rev Esp Med Quir. 2002;17:41-5.

9. Flores-Nava G, Pérez-Aguilera T, Pérez-Berbabé M. Malformaciones congénitas diagnósticadas en un hospital general. Revisión de cuatro años. Acta Pediatr Mex. 2011;32:101-6.

10. Mendieta-Alcántara G, Santiago-Alcántara E, Mendieta-Zerón H, et al. Incidencia de las cardiopatías congénitas y los factores asociados a la letalidad en niños nacidos en dos hospitales del Estado de México. Gac Med Mex. 2013:149. 
11. De Rubens-Figueroa J, Oldak-Skvirsky D, Castilla-Serna L, et al. Diagnóstico de cardiopatía congénita en en neonatos. Experiencia de 11 años en el Instituto Nacional de Pediatría. Rev Mex Cardiol. 1997;8:128-39.

12. Castillo-Espínola A, Velázquez-Ibarra A, Zetina-Solórzano $\mathrm{A}$, et al. Morbilidad posquirúrgica en pacientes pediátricos operados por cardiopatías congénitas en la UMAE de Yucatán. Arch Cardiol Mex. 2016, http://dx.doi.org/10.1016/j.acmx.2016.10.004.

13. Solano-Fiesco L, Aparicio-Osorio M, Romero-Ramírez J. Prevalencia e incidencia de cardopatías congénitas en el servicio de Cardiología Pediátrica del Hospital Central Militar, enero 2006enero 2010. Rev Sanid Milit Mex. 2015;69:171-8.

14. Varela-Ortiz J, Contreras-Santiago E, Calderón-Colmenero J, et al. Epidemiología de pacientes con cardiopatías congénitas sometidos a cirugá en un hospital privado de tercer nivel en México. Rev Invest Med Sur Mex. 2015:182-8.

15. Cervantes-Salazar J, Calderon-Colmenero J, Ramirez-Marroquin $\mathrm{S}$, et al. Pediatric cardiovascular surgical data base registry in Mexico. First report. Rev Invest Clin. 2013;65:476-82.

16. Galván-Román L, García-Hernández E, Valdespino-Estrada A, et al. Cardiopatías congénitas en el adulto diagnósticadas por ecocardiografía transtorácica. Rev Mex Cardiol. 2009;20:12-7.

17. Alva-Espinosa C. Ante el adulto con cardopatía congénita. Rev Mex Cardiol. 2006;17:155-8.

18. Vázquez-Antona C. Papel de la ecocardiografía en el adulto con cardiopatía congénita. Arch Cardiol Mex. 2002;72. S226-S32.

19. Hernández-Pacheco J, Estrada-Altamirano A, Nares-Torices M, et al. Pronóstico materno y perinatal en mujeres embarazadas con cardiopatías congénitas con circuito en el Instituto Nacional de Perinatología Isidro Espinosa de los Reyes. Perinatol Reprod Hum. 2012;28:137-46.
20. Velázquez-Torres B, Gallardo-Gaona J, Acevedo-Gallegos S, et al. Abordaje diagnóstico de la cardiopatía fetal en el Instituto Nacional de Perinatología. Ginecol Obstet Mex. 2008;76: 431-9.

21. Koerten M, Niwa K, Szatmári A, et al. Frequency of miscarriage/stillbirth and terminantions of pregnacy among women with congenital heart disease in Germany Hungary and Japan. Cir J. 2016;80:1846-51.

22. Geografía INdEy. Regiones socioeconómicas de México. 2000 [consultado $13 \mathrm{Abr}$ 2017] Disponible en: http://sc.inegi.gob.mx.

23. Knowles RL, Ridout D, Crowe S, et al. Ethnic and socioeconomic variation in incidence of congenital heart defects. Arch Dis Child. 2017;102:496-502.

24. Eslami B, Sundin O, Macassa G, et al. Gender differences in health conditions and socio-economic status of adults with congenital heart disease in a developing country. Cardiol Young. 2013;23:209-18.

25. Aubry P, Demian H. Sex differences in congenital heart disease. Ann Cardiol Angeiol (Paris). 2016;65:440-5.

26. Mackie AS, Pilote L, lonescu-Ittu R, et al. Health care resource utilization in adults with congenital heart disease. Am J Cardiol. 2007;99:839-43.

27. Briston D, Bradley E, Sabanayagam A, et al. Health Care Costs for Adults With Congenital Heart Disease in the United States 2002 to 2012. Am J Cardiol. 2016;118:590-6.

28. Bruto V, Harrison D, Fedak P, et al. Determinants of healthrelated quality of life in adults with congenital heart disease. Congenit Heart Dis. 2007;2:301-13.

29. Nelson J, Stebbins R, Strassle P, et al. Geographic distribution of live births with tetralogy of Fallot in North Carolina 2003 to 2012. Birth Defects Res A Clin Mol Teratol. 2016;106:881-7. 